\title{
Revising neutrino oscillation parameter space with direct flavor-changing interactions
}

\author{
Loretta M. Johnson ${ }^{a}$ and Douglas W. McKay ${ }^{b}$ \\ ${ }^{a}$ Department of Physics, Grinnell College, Grinnell, IA 50112; ${ }^{b}$ Department of Physics and Astronomy, University of Kansas, \\ Lawrence, KS 66045
}

\begin{abstract}
We formulate direct, neutrino flavor-changing interactions in a framework that fits smoothly with the parameterization of two- and three-state mixing of massive neutrino states. We show that even small direct interaction strengths could have important consequences for the interpretation of currently running and proposed oscillation experiments. The oscillation amplitude and the borders of the allowed regions in two- and three-flavor mixing parameter space can be sensitive to the presence of direct interactions when the transition probability is small. We use extensively the high sensitivity of the NOMAD experiment to illustrate potentially large effects from small, direct flavor violation. In the purely leptonic sector, we find that the clean $\nu_{\mu}$ and $\nu_{e}$ beams from a $\mu^{+}-\mu^{-}$ collider could provide the sharpest tests of direct flavor violation.
\end{abstract}

\section{INTRODUCTION}

The Superkamiokande collaboration's zenith angle analysis of its data [1], may sway even a skeptic to the view that neutrino oscillations have been observed. The precise form of the neutrino mass matrix and the number of neutrino species remain undetermined, however. The confusing and complicated nature of the whole collection of neutrino data in laboratory and astrophysical settings makes for an exciting playing field for workers trying to establish a "standard model" of neutrino physics. Vigorous efforts have been made to determine whether one needs only three flavors of neutrinos, mixed either two-by-two 2] or with some form of full three- state mixing [3], [4] or whether a fourth, sterile neutrino is needed in the mixture [5], [6]. There is not yet a compelling phenomenological or theoretical case for any of these reasonable, and partially successful, approaches.

Direct violation in neutrino interactions is a feature of the flavor-mixing puzzle that has received relatively little attention. Most models that predict the existence of neutrino mass and mixing, and consequently neutrino oscillations, also contain direct neutrino flavor- violating interactions. In fact, some interesting models have no neutrino masses at tree level, but flavor-violating interactions generate them in loop graphs [7], [8], [9], [10], [11]. Moreover, with the help of resonant enhancement, massless neutrinos can mix in a non-trivial way in certain models and produce flavor transitions in flight [12]. It seems natural to extend the phenomenological framework for oscillations of propagating neutrinos to include the effects of direct interactions in a way that allows one to survey all effects at once. A formalism to carry out this program is sketched and illustrated in [13], and we extend the range of applications and uncover several new features in the present paper. Specific models are not discussed here, since we emphasize the model independent features of the oscillation-plus-direct flavor violation analysis. Bounds on parameter combinations from a given model can be directly obtained from the coefficients of our effective four Fermi Lagrangian.

As in [13], our primary concern is with the accelerator experiments [14] [15] [16]. The experimental constraints on muonium-antimuonium conversion are now so tight [17] that a purely direct interaction explanation of the muon decay-at-rest (DAR) signal reported in [14 can be rather convincingly ruled out 18. Here we emphasize effects that are significant when combined with the oscillation phenomenon. For example, we show in section IV that direct effects can kill oscillations in special circumstances. We also find that in high sensitivity experiments, where tight limits in regions of $\sin ^{2} 2 \theta-\Delta m^{2}$ space are achieved, small, direct flavor violation can change a boundary by more than an order of magnitude. We illustrate this by showing examples of the effects on the large $\Delta m^{2}$, small $\sin ^{2} 2 \theta$ boundary of the $\nu_{\mu} \leftrightarrow \nu_{\tau}$ mixing set by the NOMAD collaboration [19]. In another sensitive comparison, we show the power of comparing the "wrong flavor" appearance signals from the clean $\nu_{\mu}$ and $\nu_{e}$ beams afforded by a $\mu^{+}-\mu^{-}$collider.

There are several studies where direct flavor violation is considered in the solar and atmospheric cases. In [20] the combined oscillation and direct effects are applied to an analysis of the resonant conversion of electron neutrinos to other species as the explanation of the solar neutrino deficit [21] [22] [23] [24], while in [25] and [26], an explanation using resonantly enhanced direct lepton number violation of the zenith angle effect reported in 11 was presented. In both cases significant effects were reported, though a complete explanation in terms of direct interactions is probably not possible in the solar case. The situation is not settled in the atmospheric case. A critique of alternatives to the large $\nu_{\mu^{-}} \nu_{e}$ mixing solutions to the atmospheric neutrino data such as those presented in [25], 26] and [27] is given in [28]. A model using $\nu_{\mu}$ decay that answers the objection in [28] is described in [29].

We encourage the reader interested in the impact of small, direct flavor violation on the analysis of experiments with high sensitivity to "wrong flavor" appearance to go straight to Sec. 4. The background is given in the next two sections. In the following section, the parameterization and notation are defined, and the notion of a generalized 
transition probability factor is explained. In Sec. 3 the experimental constraints on flavor violating parameters defined in Sec. 2 are summarized. As just remarked, the formalism is applied to a number of examples drawn from current or future accelerator experiments in Sec. 4. We keep the constraints on flavor-violating parameters clearly in mind in the discussion of these applications. The formalism and results of its applications are summarized and several conclusions are drawn in Secs. 5 and 6 . The general forms of the probability factors that apply to the case where $\mu$ decay provides the source of neutrinos are given in the Appendix.

\section{FORMALISM}

In this section we develop a compact parameterization of direct interaction effects in neutrino flavor-changing processes. We represent the low-energy effective interactions involving neutrinos, charged leptons and first generation quarks by the four-Fermi semileptonic (S) and leptonic (L) Lagrangians 1$]$

$$
\mathcal{L}^{S}=2 \sqrt{2} G_{F} K_{A i j}^{h}\left(\overline{l_{i}} \Gamma_{A} P_{h} U_{j a} \nu_{a}\right)\left[\bar{d} \Gamma_{A}\left(\alpha P_{L}+\beta P_{R}\right) u\right]^{\dagger}+\text { h.c. }
$$

and

$$
\mathcal{L}^{L}=2 \sqrt{2} G_{F} F_{A i j k m}^{h h^{\prime}}\left(\overline{l_{i}} \Gamma_{A} P_{h} U_{j a} \nu_{a}\right)\left(\overline{l_{k}} \Gamma_{A} P_{h^{\prime}} U_{m b} \nu_{b}\right)^{\dagger},
$$

where $U_{i a}$ is the matrix, unitary in the relativistic limit, that relates flavor as shown below in Eq.(7). Repeated indices are summed. The coefficients $\mathrm{K}$ and $\mathrm{F}$, whose indices are $\mathrm{i}, \mathrm{j}, \mathrm{k}, \ldots$, represent the coupling strengths for the different lepton flavor combinations, while the indices $\mathrm{a}, \mathrm{b}, \ldots$ label the mass eigenvalues. The coefficients $\alpha$ and $\beta$ allow for different strengths for $\mathrm{L}$ and $\mathrm{R}$ couplings to the quark currents. The Lorentz structure of the bilinear forms is labeled by $\mathrm{A}=\mathrm{S}, \mathrm{V}$ or $\mathrm{T}$ and $P_{h}$ denotes the left- and right-helicity projections. The expression in Eq.(2) is a generalization of the generic muon-decay, four- Fermi interaction to include lepton flavor violations of all types. By a Fierz transformation one can show that $F_{T}^{L L}$ and $F_{T}^{R R}$ are both identically zero. Restricting application of Eq.(2) to muon decay and (unobserved) massless neutrinos, one can show that it is not possible to test lepton number conservation from the available observables [30].

We illustrate the notation by applying it to the standard model (SM) effective, low-energy Lagrangian. The leptonic neutral-current term,

$$
\mathcal{L}_{S M}^{L N C}=\sqrt{2} G_{F}\left[\overline{l_{i}}\left(2 s_{W}^{2} \gamma_{\mu} P_{R}+\gamma_{\mu}\left(2 s_{W}^{2}-1\right) P_{L}\right) l_{i}\right]\left(\overline{\nu_{j}} \gamma^{\mu} P_{L} \nu_{j}\right),
$$

can be Fierz transformed into an equivalent charged current form

$$
\mathcal{L}_{S M}^{L N C}=\sqrt{2} G_{F}\left[\left[\overline{l_{i}}\left(2 s_{W}^{2}-1\right) \gamma_{\mu} P_{L} \nu_{j}\right]\left(\overline{\nu_{j}} \gamma^{\mu} P_{L} l_{i}\right)-2\left[\overline{l_{i}} 2 s_{W}^{2} P_{L} \nu_{j}\right]\left(\overline{\nu_{j}} P_{R} l_{i}\right)\right] .
$$

Next we add the SM leptonic charged-current effective Lagrangian

$$
\mathcal{L}_{S M}^{L C C}=2 \sqrt{2} G_{F}\left[\overline{l_{i}} \gamma_{\mu} P_{L} \nu_{i}\right]\left(\overline{\nu_{j}} \gamma^{\mu} P_{L} l_{j}\right)
$$

and the semileptonic effective Lagrangian

$$
\mathcal{L}_{S M}^{S}=2 \sqrt{2} G_{F}\left[\bar{u} \gamma_{\mu} P_{L} d\right]\left(\overline{l_{j}} \gamma^{\mu} P_{L} \nu_{j}\right)+\text { h.c. }
$$

The F and K coefficients can now be read off from the SM effective low energy Lagrangian:

\begin{tabular}{|l|l|l|l|l|}
\hline$K_{V j j}^{L}$ & $F_{V i i j j}^{L L}(i \neq j)$ & $F_{V i i i i}^{L L}$ & $F_{V i j j i}^{L L}(i \neq j)$ & $F_{S i j i j}^{L R}$ \\
\hline 1 & 1 & $\left(s_{W}^{2}+1\right) / 2$ & $\left(2 s_{W}^{2}-1\right) / 2$ & $-2 s_{W}^{2}$ \\
\hline
\end{tabular}

\footnotetext{
${ }^{1}$ The CKM factors multiplying $G_{F}$ play no direct role in our discussion, so they are suppressed in the notation.
} 


\section{A. Lepton Flavor-Changing Transitions}

In the usual analysis of lepton flavor oscillations, the neutrinos are treated as massless in the matrix element kinematics. Approximating the plane wave phase factors for the propagating neutrino to leading order in the masses, one factors out the transition amplitude to write

$$
\left\langle\nu_{j}(t) \mid \nu_{i}(0)\right\rangle=\sum_{a}\left\langle\nu_{j} \mid \nu_{a}\right\rangle e^{-i m_{a}^{2} t / 2 E}\left\langle\nu_{a} \mid \nu_{i}\right\rangle=\sum_{a} U_{j a}^{*} e^{-i m_{a}^{2} t / 2 E} U_{i a} .
$$

However, this factorization is not valid when the neutrino masses are taken into account [31]. Though we work in the ultra-relativistic limit, where the neutrino masses can be ignored in the arguments of the matrix elements, we find the process dependence as discussed in 31 to be a useful setting for the intermediate stages of our development. We generalize the SM weak-process initial states to include new physics; for example,

$$
\left|\nu_{\mu}\right\rangle_{W P} \sim \sum_{a}\left|\nu_{a}\right\rangle\left\langle\nu_{a}, \mu^{+}\left|\mathcal{L}_{S M}^{S}\right| \pi^{+}\right\rangle \rightarrow \sum_{a}\left|\nu_{a}\right\rangle\left\langle\nu_{a}, \mu^{+}\left|\mathcal{L}^{S}\right| \pi^{+}\right\rangle,
$$

with $L^{S}$ defined in Eq.(1). Similarly, we create a weak-process final state for the detector and use it with Eq.(8) to construct the transition amplitude. More generally, denoting initial and final states by $\left|I^{s, d}\right\rangle$ and $\left|F^{s, d}\right\rangle$, we define

$$
\mathcal{M}_{a}^{s}=\left\langle F^{s}\left(\nu_{a}\right)\left|\mathcal{L}^{S}+\mathcal{L}^{L}\right| I^{s}\right\rangle
$$

and

$$
\mathcal{M}_{a}^{d}=\left\langle F^{d}\left|\mathcal{L}^{S}+\mathcal{L}^{L}\right| I^{d}\left(\nu_{a}\right)\right\rangle
$$

as the source and detector transition matrix elements involving a mass eigenstate of $\nu_{a}$. We can write the full transition amplitude, including direct new interactions, from creation to detection of the neutrino as

$$
\left\langle\nu_{j}(t) \mid \nu_{i}(0)\right\rangle_{N I}=\sum_{a}\left\langle\nu_{j} \mid \nu_{a}\right\rangle e^{-i m_{a}^{2} t / 2 E}\left\langle\nu_{a} \mid \nu_{i}\right\rangle_{N I}=\mathcal{M}_{a}^{d} e^{-i m_{a}^{2} t / 2 E} \mathcal{M}_{a}^{s},
$$

where a sum over the label, a, of mass eigenstates is implicit here and in what follows, and $N I$ indicates that new interactions are included.

As an example, let us consider a SM process that is a background to electron appearance experiments ${ }^{\beta}$, namely $\pi^{+} \rightarrow e^{+} \nu_{e}$ followed by $\nu_{e} N_{i} \rightarrow e^{-} N_{f}$, where $N_{i, f}$ designate inital and final hadronic (nuclear) states. We expand the SM to include the possibility of neutrino oscillation for illustration. The transition matrix elements for the source and detector processes are

$$
\mathcal{M}_{a}^{s}=2 \sqrt{ } 2 G_{F} K_{V 11}^{L}\left\langle e^{+} \nu_{e}\left|\left(\bar{l}_{1} \gamma_{\mu} P_{L} U_{1 a} \nu_{a}\right)^{\dagger}\right| 0\right\rangle\left\langle 0\left|\mathcal{O}_{V}^{\mu}\right| \pi^{+}\right\rangle
$$

and

$$
\mathcal{M}_{a}^{d}=2 \sqrt{ } 2 G_{F} K_{V 11}^{L}\left\langle N_{f}\left|\mathcal{O}_{V}^{\mu \dagger}\right| N_{i}\right\rangle\left\langle e^{-}\left|\bar{l}_{1} \gamma_{\mu} P_{L} U_{1 a} \nu_{a}\right| \nu_{e}\right\rangle
$$

where $\mathcal{O}_{V}^{\mu}$ designates the quark current operator $\bar{d} \gamma^{\mu} P_{L} u$ appropriate to the SM in Eq.(1). In the following, we will continue to use $\mathcal{O}$ to designate the hadronic current operator. In the ultra-relativistic limit, the neutrino masses are set equal to zero in the spinors and only the leading phase dependence on masses is kept. The transition amplitude squared can then be factorized into a SM product of matrix elements squared times the oscillation probability, involving a sum over mass eigenstates:

$$
\left|\mathcal{M}_{a}^{d} e^{-i m_{a}^{2} t / 2 E} \mathcal{M}_{a}^{s}\right|^{2}=\left(2 \sqrt{ } 2 G_{F}\right)^{4}\left|\left(\bar{e} \gamma_{\mu} P_{L} \nu\right)^{*}\left(\bar{e} \gamma_{\lambda} P_{L} \nu\right)\left\langle 0\left|\mathcal{O}_{V}^{\mu}\right| u \bar{d}\right\rangle\left\langle N_{f}\left|\mathcal{O}_{V}^{\lambda \dagger}\right| N_{i}\right\rangle\right|^{2}\left|U_{1 a}^{*} e^{-i m_{a}^{2} t / 2 E} U_{1 a}\right|^{2},
$$

where we have used the fact that $K_{V 11}=1$ in the SM. Schematically we can write the above as

$$
\left|\mathcal{M}_{a}^{d} e^{-i m_{a}^{2} t / 2 E} \mathcal{M}_{a}^{s}\right|^{2} \sim \Phi_{e} \sigma\left(\nu_{e} N_{i} \rightarrow e^{-} N_{f}\right) P_{e \rightarrow e}
$$

In Eq.(15) $\Phi_{e}$ designates the $\nu_{e}$ flux, $\sigma\left(\nu_{e} N_{i} \rightarrow e^{-} N_{f}\right)$ the SM electron neutrino charged current cross section and $P_{e \rightarrow e}=\left|U_{1 a}^{*} e^{-i m_{a}^{2} t / 2 E} U_{1 a}\right|^{2}$ designates the probability that an electron neutrino produced at the source appears as an electron neutrino at the target. Our next task is to achieve an equally transparent factorization that includes direct violation of lepton flavor.

\footnotetext{
${ }^{2}$ In the ultra-relativistic limit, one may take $\mathrm{t}=\mathrm{L}$, the propagation length, in the following expressions.

${ }^{3}$ These are experiments that look for signals of neutrino flavor $\mathrm{j}$ interactions in a beam of neutrinos created with flavor $\mathrm{i} \neq \mathrm{j}$.
} 


\section{B. Lepton Flavor-Changing Probability Factor}

To identify a flavor-changing factor that includes direct flavor violation effects and plays the same role as the oscillation probability factor $P_{e \rightarrow e}$ in Eq.(15), we use the amplitudes defined in Eqs.(9), (10) and (11) and follow the pattern of our preceding discussion of Eqs.(12) - (15). Our new "probability" factor should reduce to the oscillation probability when the direct flavor-violating couplings are turned off, of course. For this purpose, we continue to use the pion decay source and nucleon/nucleus detector example, but now we will extract a lepton appearance probability factor from combined oscillations and direct interactions. We adopt an illustrative model with a V-A current with $K_{V 2 l}^{L} \neq 0$, with $l=\tau$ or e but with all other non-SM coefficients $K_{A i j}^{h}=0$. This model produces a $\pi^{+} \rightarrow \mu^{+}+\nu_{l}$ transition at the source but leaves only the SM process $\nu_{l}+N_{i} \rightarrow l^{-}+N_{f}$ active at the target. We shall refer to this as a "source-only" situation. The electron production rate at the detector is proportional to

$$
\left|\mathcal{M}_{a}^{d} e^{-i m_{a}^{2} t / 2 E} \mathcal{M}_{a}^{s}\right|^{2}=\left|\left\langle l^{-} N_{f}\left|\mathcal{L}^{S}\right| \nu_{a} N_{i}\right\rangle e^{-i m_{a}^{2} t / 2 E}\left\langle\mu^{+} \nu_{a}\left|\mathcal{L}^{S}\right| \pi^{+}\right\rangle\right|^{2}
$$

where the right-hand side, after spelling out the terms from Eq.(1) and taking the ultra-relativistic limit, reads

$$
\left(2 \sqrt{ } 2 G_{F}\right)^{4}\left|\bar{l} \gamma_{\lambda} P_{L} \nu\left\langle N_{f}\left|\mathcal{O}_{V}^{\lambda}\right| N_{i}\right\rangle\right|^{2}\left|\left\langle 0\left|\mathcal{O}_{V}^{\sigma}\right| \pi^{+}\right\rangle \bar{\nu} \gamma_{\sigma} P_{L} \mu\right|^{2}\left|\left(U_{2 a}+K_{V 2 l}^{L} U_{l a}\right) e^{-i m_{a}^{2} t / 2 E} U_{l a}^{*}\right|^{2},
$$

where $K_{V 11}=1$ has been used. The $\nu_{l}$ appearance probability factor, including the direct flavor- violation coefficient $K_{V 2 l}^{L}$, is given by the final expression within absolute magnitude signs in Eq.(17). The first term in this expression is the usual oscillation amplitude for transition from $\nu_{\mu}$ to $\nu_{l}$ between source and detector.

To make the notation more compact in the following discussion, we define

$$
\begin{aligned}
\left(m_{b}^{2}-m_{a}^{2}\right) t / 2 E & \equiv 2 x \\
K_{V 2 l}^{L} & \equiv \tan \psi e^{2 i \phi},
\end{aligned}
$$

where the choices of $\mathrm{a}$ and $\mathrm{b}$ in the definition of $\mathrm{x}$ and the values of $\psi$ and $\phi$ depend upon the situation, and we will illustrate the two-flavor mixing and the three-flavor mixing with dominance by one mass scale [3]. We have chosen the parametrization of $K_{V 2 l}^{L}$ by tan $\psi$ to emphasize that the flavor-violating amplitude could, in principle, be larger than the flavor-conserving one. In practice it is constrained by experiment to be small compared to the standard model amplitude, but the parametrization reminds us that the direct flavor- violating amplitude cannot be represented as a unitary rotation to a "source basis" except in the small tan $\psi$ limit with $\phi$ negligible. In addition, the special circumstance must occur that the effective flavor-violating four-Fermi interactions have a V-A structure to match the SM structure. Nonetheless, we will refer to the transition factor modulus squared as a probability or probability factor in what follows. As we show below, the survey of direct effects on the standard two- and three-family mixing plots with $\Delta m^{2}$ vs. $\sin ^{2}(2 \theta)$ contours or $\tan ^{2} \theta_{13}$ vs. $\tan ^{2} \theta_{23}$ for fixed $\delta m^{2}$, for example, can be straightforwardly extended to include direct lepton number violation.

Isolating the probability factor in Eq.(17), we write

$$
P_{\mu \rightarrow l}=\left|U_{2 a} e^{-i m_{a}^{2} t / 2 E} U_{l a}^{*}+\tan \psi e^{2 i \phi} U_{l b} e^{-i m_{b}^{2} t / 2 E} U_{l b}^{*}\right|^{2},
$$

no sum on $l$, which labels e or $\tau$. With $\phi=0$, in "mock" unitary form, f we have

$$
P_{\mu \rightarrow l}=\left|\cos \psi U_{2 a} e^{-i m_{a}^{2} t / 2 E} U_{l a}^{*}+\sin \psi U_{l b} e^{-i m_{b}^{2} t / 2 E} U_{l b}^{*}\right|^{2} /(\cos \psi)^{2}
$$

and, approximating the overall $(\cos \psi)^{-2}$ factor by 1 to order $\psi^{2}$, Eq.(19) can also be recast as

$$
P_{\mu \rightarrow l} \simeq\left|V_{22} U_{2 a} e^{-i m_{a}^{2} t / 2 E} U_{l a}^{*}+V_{2 l} U_{l b} e^{-i m_{b}^{2} t / 2 E} U_{l b}^{*}\right|^{2} .
$$

We have identified $V_{22}=\cos \psi$ and $V_{2 l}=\sin \psi$ in Eq.(20), which suggests the interpretation that the $\mu$ from $\pi$ decay is accompanied by a $\nu_{\mu}$ with amplitude $V_{22}$ and followed by propagation and an oscillation to $\nu_{e}$ with amplitude $U_{2 a} e^{-i m_{a}^{2} t / 2 E} U_{1 a}^{*}$ or is accompanied by a $\nu_{l} l \neq \mu$ with amplitude $V_{2 l}$ and then followed by $\nu_{l}$ propagation with amplitude $U_{l b} e^{-i m_{b}^{2} t / 2 E} U_{l b}^{*}$ to remain $\nu_{l}$. This picture, though only approximately valid and only then in the circumstances described above, is useful for seeing how the direct lepton flavor violations can be worked in with the oscillation between flavors in a reasonably seamless fashion.

\footnotetext{
${ }^{4}$ For present purposes, we assume $\phi$ is constrained to be small by experimental limits on CP violation.
} 


\section{CONSTRAINTS ON THE STRENGTHS OF DIRECT LEPTON-FLAVOR VIOLATIONS}

The identities of electron neutrino, muon neutrino and tau neutrino are preserved to a good accuracy in decays and collisions. Within neutrino data alone, the constraints on the sizes of lepton-flavor violations, though tight, often leave room for violations at the $1 / 2 \%$ level in cross-sections and rates. In the realm of purely charged lepton processes, on the other hand, the high degree of experimental control has lead to fantastically small limits on some of the ratios of lepton-number- violating to lepton-number-preserving interaction strengths. In this section we briefly survey the constraints that affect our analysis the most directly. The considerations presented here govern our choice of parameter values in the following sections as we illustrate some of the possible effects that direct interactions produce in concert with oscillations.

Weak SU(2) invariance of any new physics interaction Lagrangian that produces lepton number violation will generally relate the purely leptonic processes to those involving neutrinos [18]. The severe experimental limits on the kinematically allowed leptonic processes then translate into limits on processes involving leptons and neutrinos, up to group theory factors and $\mathrm{SU}(2)$-violating mass-splittings among members of boson multiplets that mediate the lepton number violations. The purely leptonic processes whose experimental limits impose the strictest bounds on lepton number violation in muon-source experiments are muonium to antimuonium conversion [17], $\mu \rightarrow e e e, \tau \rightarrow \mu e e$ and $\tau \rightarrow \mu \mu e$ [32] . There are similar constraints [32] on the strengths of semileptonic processes violating lepton number that follow from $\tau \rightarrow \pi+e, \tau \rightarrow \pi+\mu$ and from $\mu$ conversion to e on Ti nuclei [33]. The constraints are summarized in Table 1. The "experimental constraints" shown in Table 1 are actually those that apply to the charged-lepton processes listed. The relationship to the bounds on the lepton-number-violating coefficients for the neutrino processes is somewhat indirect, since group theory factors, ratios of masses of virtual bosons mediating the processes and effects due to differences between, say, S and V structure must be included. Allowing a possible factor of two from ClebschGordon coefficients and a generous factor of two in the ratio of masses of exchange bosons within the same $\mathrm{SU}(2)$ multiplet, the coefficients shown in Table 1 are bounded by roughly eight times the purely charged- lepton process limits listed there [18]. This value is given in the column labeled "model independent constraint". The precise value of the factor for a given process is model dependent. In Table 1 and in the rest of the paper, a superscript $\mathrm{L}$ is to be understood if none is shown explicitely.

\begin{tabular}{l|l|l|l}
\hline \hline coefficient & process & experimental constraint & model independent constraint \\
\hline$F_{V 2111}$ & $\mu \rightarrow e e e$ & $1.0 \times 10^{-6}$ & $8.0 \times 10^{-6}$ \\
$F_{V 2112}$ & muonium-antimuonium & $3.0 \times 10^{-3}$ & $2.4 \times 10^{-2}$ \\
$F_{V 2113}$ & $\tau \rightarrow \mu^{+} e e$ & $2.9 \times 10^{-3}$ & $2.3 \times 10^{-2}$ \\
$F_{V 2213}$ & $\tau \rightarrow e^{-} \mu \mu$ & $3.3 \times 10^{-3}$ & $2.6 \times 10^{-2}$ \\
$F_{V 2311}$ & $\tau \rightarrow \mu^{-} e e$ & $3.2 \times 10^{-3}$ & $2.5 \times 10^{-2}$ \\
$F_{V 2312}$ & $\tau \rightarrow e^{+} \mu \mu$ & $2.9 \times 10^{-3}$ & $2.3 \times 10^{-2}$ \\
$K_{V 21}$ & $\mu-e$ conversion & $1.8 \times 10^{-7}$ & $1.5 \times 10^{-6}$ \\
$K_{V 31}$ & $\tau \rightarrow e \pi^{0}$ & $8.2 \times 10^{-3}$ & $6.6 \times 10^{-2}$ \\
$K_{V 32}$ & $\tau \rightarrow \mu \pi^{0}$ & $8.5 \times 10^{-3}$ & $6.8 \times 10^{-2}$ \\
\hline \hline
\end{tabular}

TABLE I. Limits on charged lepton processes and the corresponding neutrino process coefficients $\mathrm{F}$ and K. The model independent constraints on the $\mathrm{F}$ and $\mathrm{K}$ coefficients are taken to be roughly eight times the charged lepton process limits. For $\mathrm{K}$ constraints we take $\mathrm{h}=\mathrm{L}, \alpha=1$ and $\beta=0$ in Eq.(1). 


\section{IMPACT ON ANALYSIS OF APPEARANCE EXPERIMENTS}

The very term "neutrino oscillation" implies path-length-dependent variation of the probability that a given flavor of neutrino appears in the beam. Moreover, neutrino oscillation and neutrino mass are so tightly linked that evidence for the former is considered tantamount to proof of the latter - certainly in the case of vacuum oscillations. Conversely, the absence of oscillations in a neutrino flavor-violating effect is tantamount to elimination of neutrino mass as an explanation of its origin. This is not necessarily so when flavor violation is expanded to include direct interactions. This point is among a number that we make in the present section. The examples chosen are all consistent with the bounds described in the previous section and summarized in Table 1.

At the beginning of this section we take the neutrino source to be $\pi$ - decay. We explore the interplay in the $\nu_{\mu} \leftrightarrow \nu_{\tau}$ case between the mass-induced, oscillating amplitude and the directly-induced, non-oscillating amplitude. While it is true that small masses generally lead to vacuum oscillations, it is not strictly true that the absence of oscillations proves that the neutrinos are massless. In a sense this is a complement to the well-known result that massless neutrinos can oscillate as they pass through matter. These effects are shown in Figs. 1. and 2. In Fig. 3., we show the result of including the flavor-violation parameter "axis", $\psi$, in the analysis of the probability bounds in the sensitive NOMAD experiment. The result is dramatic. Then we make the point that the bound on the oscillation mixing angle for fixed $\Delta m^{2}$ depends in general on the value of the direct flavor violation parameter $\psi$. This is shown in Fig. 4, which includes the possibility that the same direct flavor violation occurs at both the source and the detector. This discussion is followed by the expansion of the analysis to the three flavor situation. Figures 5 . and 6 . show how different $\tan ^{2} \theta_{13}-\tan ^{2} \theta_{23}$ boundaries appear as different fixed $\psi$ and $\Delta m^{2}$ "slices" of the parameter space are taken. Figure 5. is appropriate to the upcoming MiniBooNe experiment, while Fig. 6. applies to the reported NOMAD probability bound.

In the last part of this section, we show the power of the clean $\nu_{\mu}$ and $\nu_{e}$ beams from proposed $\mu^{+} / \mu^{-}$storage rings to make sensitive tests for direct flavor violation in the purely leptonic sector. Of particular note is the prospect of advancing another order of magnitude into the parameter space of direct $\nu_{e} \leftrightarrow \nu_{\mu}$ flavor violations.

\section{A. $\pi$-decay as the Neutrino Source}

Restricting ourselves at first to mixing of two mass eigenstates, we write Eq.(19) for the probability of lepton appearance in the pion decay as the source in an "all angles" form and defining $F_{V 2 l}^{L}=\tan \psi e^{2 i \phi}$

$$
P_{\mu \rightarrow l}=\left|\cos \psi\left(-\cos \theta \sin \theta e^{i x}+\sin \theta \cos \theta e^{-i x}\right)+\sin \psi e^{2 i \phi}\left(\cos ^{2} \theta e^{i x}+\sin ^{2} \theta e^{-i x}\right)\right|^{2} \cos ^{-2} \psi .
$$

As defined above, $x=\left(m_{2}^{2}-m_{1}^{2}\right) t / 4 E$ in applicaton to Eq.(21), and the two-flavor mixing matrix is written as

$$
U=\left(\begin{array}{cc}
\cos \theta & \sin \theta \\
-\sin \theta & \cos \theta
\end{array}\right)
$$

Rearranging terms and consolidating them, we arrive at the rather transparent form of Eq.(21)

$$
P_{\mu \rightarrow l}=\tan ^{2} \psi+\frac{\sin 2 \theta \sin 2(\theta-\psi) \sin ^{2} x}{\cos ^{2} \psi}+4 \tan \psi \sin 2 \theta \sin \phi \sin x(\cos 2 \theta \sin \phi \sin x-\cos \phi \cos x) .
$$

Equation (23) has the obvious and expected feature that if $\psi=0$, we have $P_{\mu \rightarrow l}=\sin ^{2} 2 \theta \sin ^{2} x$, the usual two-flavor, pure mass-mixing, oscillation formula in terms of the mixing angle $\theta$ and the factor $x=\Delta m^{2} L / 4 E$. Equally obvious and expected is the relationship $P_{\mu \rightarrow l}=\tan ^{2} \psi$ that holds when $\theta=0$ or $x=0$. What is not expected is that, when $\sin \phi=0, P_{\mu \rightarrow l}=\tan ^{2} \psi$, independent of $x$, when $\theta-\psi=\pi n / 2$, where $\mathrm{n}$ is an integer. Looking back at Eq.(21), we see that when $\theta-\psi=\pi n / 2$ and when $\phi=0$ the coefficient of $e^{i x}$ from the $\nu_{\mu} \rightarrow \nu_{l}$ oscillation cancels against its coefficient from the $\nu_{l} \rightarrow \nu_{l}$ term in the direct flavor violation amplitude. The remaining overall phase from the $e^{-i x}$ factor disappears in the modulus squared and one is left with simply $P_{\mu \rightarrow l}=\tan ^{2} \psi=\tan ^{2} \theta$. This somewhat surprising result in the case where there is direct flavor violation in the DIF source dramatizes the implications of Eq.(23) for interpreting signals for oscillation, or lack thereof, in variable baseline experiments. There are counterparts to this source effect in the muon decay case [14] [16] as well as in the cases where direct flavor violation occurs only at the detector or in both the detector and the source. We will comment further on these situations and on three-flavor mixing below.

The condition for exact cancellation of the $\mathrm{L} / \mathrm{E}$ dependence is unlikely, of course, but the interplay between the $\psi$ and $\theta$ dependence is generic, and it affects, possibly radically if mixing angles are small, the interpretation of signals 
that show variation with path length. We illustrate the L/E-dependent effects caused by the interference between the pure oscillation term and the direct flavor violation in Figs. 1 and 2.

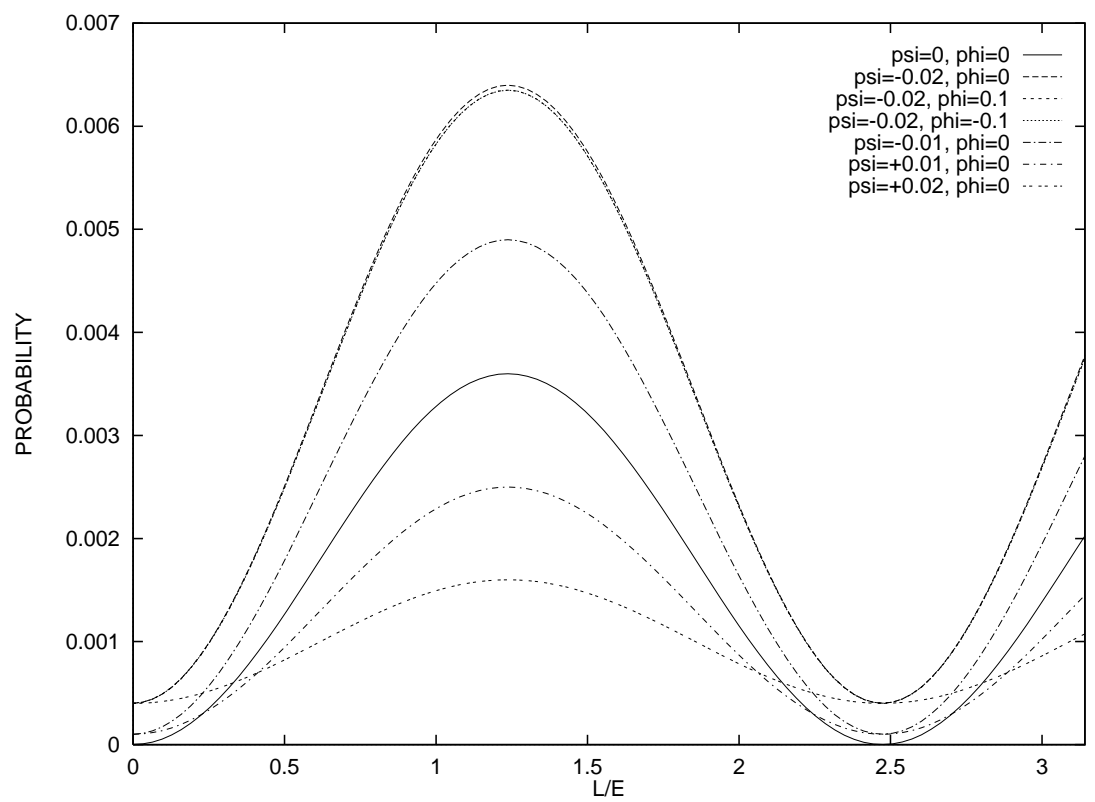

FIG. 1. Dependence of $P_{\mu \rightarrow l}$ on $x$ for $\theta=0.03$ and several different values of $\psi$ and with $\Delta m^{2}=1 e v^{2}$. A Gaussian smearing model has been adopted with $\sigma=0.016$, appropriate to the NOMAD experiment, which we will use as an illustrative example. The top three curves, which are barely distinguishable from one another, show the very weak dependence on the CP-violating phase $\phi$

Figure 1 illustrates the variation of $P_{\mu \rightarrow l}$ with L/E for fixed $\theta=0.03$, or $\sin ^{2} 2 \theta=0.0036$, for various values of $\psi$. Figure 2 shows the variation for $\psi=-0.02$ and various values of $\theta$. Several combinations of $\theta$ and $\psi$ can lead to a given curve, which suggests that it would not be straightforward to disentangle the oscillation parameters from the comparison of the $x$-dependence of the probability with data if small direct effects were included in the analysis.

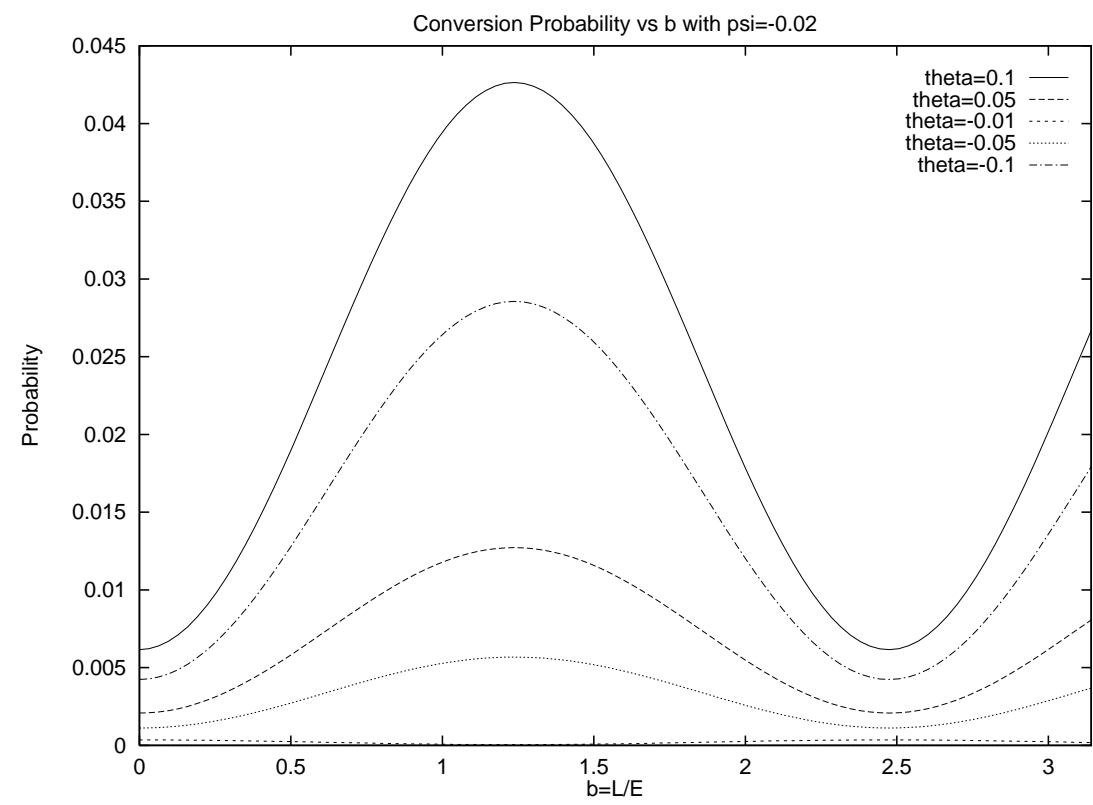

FIG. 2. Dependence of $P_{\mu \rightarrow l}$ on $x$ for $\psi=-0.02$ and several different values of $\theta$ and with $\Delta m^{2}=1 e v^{2}$.

For completeness, we also show in Fig. 1 the (weak) influence of $\phi$ on the two-flavor oscillation amplitude. Comparing the top three curves, we see that including the $\phi$-dependent effects for reasonable $\phi$ values leaves the picture essentially unchanged, as one expects when a small CP-violating phase rests upon a small flavor-violating amplitude. 
A CP-violating phase of order one is needed if it is to make an observable impact. We reserve the analysis of effects from large CP-violating phases [34] for a future study.

We see that the behavior of the probability is governed essentially by the second term in Eq. (23). This term makes it clear that even small $\psi$ values can have a large effect if the mixing angle $\theta$ is of the same order of magnitude as $\psi$.

Are there circumstances where the sizes of these direct effects could be big enough to be observable? A glance at Table 1 shows that $K_{V 21}(=\tan \psi)$ is constrained to be far too small, even with generous allowance for SU(2) breaking effects, to modify the pure oscillation picture of $\nu_{\mu}$ from $\pi$ decay oscillating to $\nu_{e}$. The $K_{V 32}$ bound is much looser, however, and interesting effects could occur in $\nu_{\mu} \rightarrow \nu_{\tau}$ two-flavor mixing with tan $\psi \leq 0.02$ if $\theta_{\mu \tau}$ were of the order of 0.1 or less. A large mixing angle is required between $\nu_{\mu}$ and another species by the two-flavor fit to the atmospheric neutrino anomaly. Thus mixing with a sterile neutrino is required, in order to have a direct interaction effect that shows up in a (weakly) mixed $\nu_{\mu} \leftrightarrow \nu_{\tau}$ sector and significantly modifies the $\nu_{\tau}$ appearance signal from a pion decay source of $\nu_{\mu}$. For small appearance probabilities, the change in signal depends rather sensitively on the value of the direct interaction strength. We give some detail in an example in the $\mu$ decay case in subsection B below.

\section{Impact on Boundaries in $\mu-\tau$ Mixing Space}

Failure to detect $\nu_{\tau}$ 's in an appearance search allows one to set confidence level curves in $\mu-\tau$ mixing space, and this gives another slant on the application of our formalism. The recent NOMAD results [19] give the smallest probability, and tightest large $\Delta m^{2}$ limits on the allowed region of parameter space for two-flavor, $\nu_{\mu} \leftrightarrow \nu_{\tau}$ mixing. To illustrate the impact of small direct interaction effects on the NOMAD bound, we approximate their boundary curve, which corresponds to a $\mathrm{P}=0.0006$ appearance probability, by the simple Gaussian smearing model [32] with parameters fit to reproduce the main features of the NOMAD boundary in $\sin ^{2} \theta$ vs. $\Delta m^{2}$ plane for mixing of $\nu_{\mu}$ and $\nu_{\tau}$. Keeping these parameters fixed, we replot the contour in the $\sin ^{2} 2 \theta-\Delta m^{2}$ plane for several small values of $\psi$. As before the small $\phi$ effects are not interesting for our present point, and we set $\phi=0$. The result is shown in Fig. 3 .

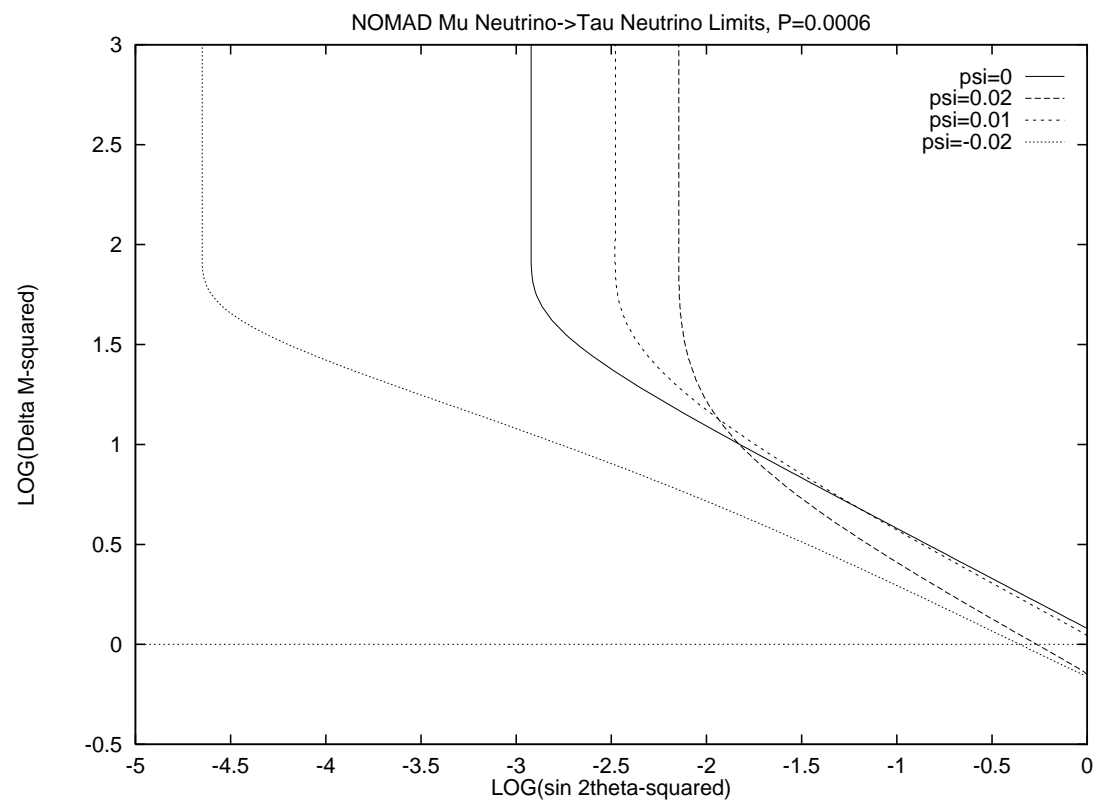

FIG. 3. Model of the NOMAD 90\% C.L. limit on $\nu_{\mu} \rightarrow \nu_{\tau}$ oscillations for several values of $\psi$ with $\phi=0$. The curves correspond to $\mathrm{P}=0.0006$.

The boundaries of the null search results are significantly revised even when small direct effects, consistent with the bounds from other experiments, are introduced. NOMAD's high sensitivity to small appearance probability in the large $\Delta m^{2}$ region is the reason that the inclusion of small direct amplitudes has such a pronounced effect.

A few comments on the shape of the different curves is in order. NOMAD has a small average L/E value, so it is sensitive only to large $\Delta m^{2}$. Their L/E distribution is broad, so the smearing almost completely damps the oscillations in the boundary curve at large $\Delta m^{2}$. The Gaussian model is even more extreme in this respect than the actual fit to the data. Because of the factor $\sin 2(\theta-\psi)$ in the expression for $P_{\mu \rightarrow \tau}$, for $0 \leq \theta \leq \pi / 2$ the positive $\psi$ values reduce the amplitude and the $\sin ^{2} x$ factor has to compensate. This eventually drives the curve to higher $\Delta m^{2}$ for a given $\theta$ value. The opposite behavior occurs when $\psi$ is negative. The role of the term $\tan ^{2} \psi$ is most apparant at 
large values of $\theta$, where the $\psi$ in the argument of sine is inconsequential, but the addition of $\tan ^{2} \psi$ to the probability allows a fit with somewhat smaller $\Delta m^{2}$ at $\theta=\pi / 2$. The crossing of positive $\psi$ curves with the $\psi=0$ curve is forced by the large $\Delta m^{2}$ and small $\Delta m^{2}$ behaviors just described.

\section{Direct Flavor-Change Bounds in $\psi-\theta$ Space}

Proposed high-sensitivity experiments to probe smaller $\Delta m^{2}$ and $\sin ^{2} 2 \theta$ regions can also place discovery limits and upper bounds on direct, flavor-violating interactions involving neutrinos. For example, Fermilab [35] and CERN [36] proposals aim to push $P_{\mu \rightarrow e}$ bounds down to $10^{-4}$ at $90 \%$ C.L., while the Fermilab-Soudan experiment, MINOS [38], is shooting for a bound of $10^{-2}$ on $P_{\mu \rightarrow \tau}$. Similar sensitivity is proposed in muon collider sources of pure $\nu_{e}$ and $\nu_{\mu}$ beams.

Let us consider the situation where $\nu_{\mu}$ 's originate from $\pi$ decays, which will be the case in the MiniBooNE experiment [35]. If the new flavor physics is only at the source, Eq.(23) applies. If one has the same new physics amplitude at the source and detector, the corresponding expression is

$$
P_{\mu \rightarrow l}=4 \tan ^{2} \psi \cos ^{2} x+\sin ^{2} 2 \theta \sin ^{2} x-2 \tan \psi \sin 2 \theta \sin 2 \phi \sin 2 x .
$$

Assuming that the $\mathrm{CP}$-violating phase, $\phi$, is small, we can readily illustrate the influence of the $\mathrm{x}$ and $\theta$ values on the limits on $\psi$ imposed by a given $90 \%$ C.L. bound on $P_{\mu \rightarrow l}$. In Fig. 4 we show the boundaries in $\psi-\theta$ space for a fixed value of $\mathrm{x}=1.27(\mathrm{~L} / \mathrm{E}) \Delta m^{2}=1$ when $P_{\mu \rightarrow l} \leq 10^{-4}$ is imposed. Fig 4 gives the source-only case and the source-plus-detector case boundaries. The specifications of MiniBooNE are $L \simeq 500 \mathrm{~m}$ and typically $0.5 \leq \mathrm{E} \leq 1.0 \mathrm{GeV}$ [35], so $\mathrm{x} \simeq \Delta m^{2}$ for purposes of translating the graphs to MiniBooNE's capabilities.

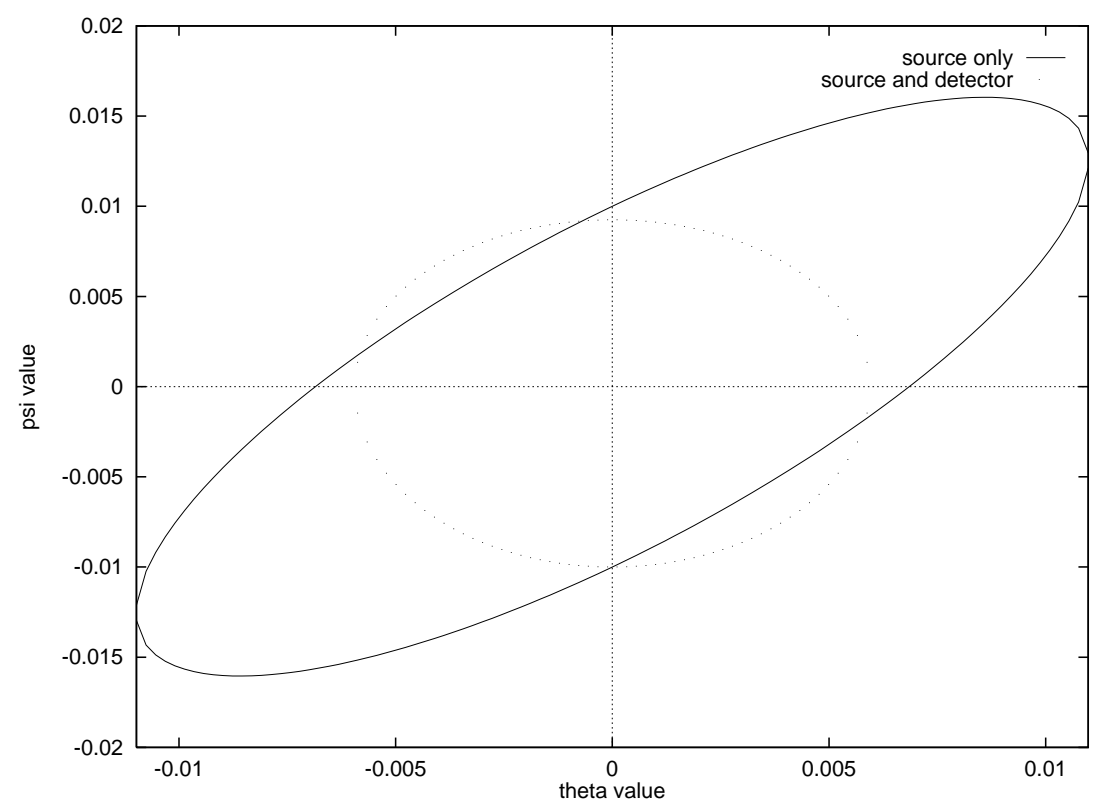

FIG. 4. Contours of $90 \%$ C.L. upper bounds in $\psi-\theta$ space for $\mathrm{x}=1$ and $P_{\mu \rightarrow e}=10^{-4}$. The cases where the new physics is at the source only and where it is at both the source and detector with equal amplitudes are shown.

In Fig. 4 the areas within the "egg-shaped" regions are allowed at the 90\%C.L., and the maximum allowed direct flavor violation parameter is evidently a function of both $\theta$ and $\mathrm{x}$. It is generally assumed that the best bound on the flavor-violating amplitudes is obtained when the oscillations are not present, but the "tilted egg" in Fig. 4 shows that this is not necessarily the case. The interference between the oscillation and direct amplitudes in the sourceonly situation makes the biggest direct flavor violation effect occur at non-zero $\theta$. When the same flavor violation is assumed to apply at the source and the detector, the symmetry of the set-up ensures that the maximum allowed value of $\psi$ occurs at $\theta=0$, which is the usual expectation.

With the $\Delta m^{2}$ value chosen, the bound $|\psi| \leq 1.6 \times 10^{-2}$ results, which is an order of magnitude stronger than the current direct bound from neutrino processes, but still much weaker than the bound that can be inferred from the limits on $\mu \rightarrow e$ transitions in heavy nuclei as listed in Table I. 
Distinct new possibilities are created when e, $\mu$ and $\tau$-flavors are all included in the picture with mixing plus direct flavor violation. Here we re-examine the $\pi$-source, e and $\tau$ appearance possibilities with three flavors, using the "one mass-scale-dominance" model [3].

Since the experimental constraint on $K_{V 21}$ makes it irrelevant for our purposes (see Table 1), we do not include it in the expressions below. With flavor violation at the source, and restricting ourselves to V-A structure, we need only consider the $K_{V 22}$ and $K_{V 23}$ coefficients. To the first order in flavor violation, we have

$$
P_{\mu \rightarrow e}=\sin ^{2} 2 \theta_{13} \sin ^{2}\left(\theta_{23}+\psi_{\mu}\right) \sin ^{2} x \cos ^{-2} \psi_{\mu}
$$

and

$$
P_{\mu \rightarrow \tau}=\tan ^{2} \psi_{\mu}+4 \sin ^{2} x \cos ^{2} \theta_{13} \cos \theta_{23} \cos ^{-2} \psi_{\mu} \sin \left(\theta_{23}+\psi_{\mu}\right)\left(-\sin \psi_{\mu}+\cos ^{2} \theta_{13} \cos \theta_{23} \sin \left(\theta_{23}+\psi_{\mu}\right)\right),
$$

where $K_{23} \equiv \tan \psi_{\mu}$ and the mixing-angle convention is that of [32]; namely $U_{13}=\sin \theta_{13} e^{-\delta_{13}}, U_{23}=\sin \theta_{23} \cos \theta_{13}$ and $U_{33}=\cos \theta_{13} \cos \theta_{23}$. We have assumed $\delta_{13}=0$ and real $K_{V i j}$ values. $P_{\mu \rightarrow e}\left(P_{\mu \rightarrow \tau}\right)$ includes the amplitude that $\nu_{\mu}$ is produced at the source and oscillates to $\nu_{e}\left(\nu_{\tau}\right)$ plus the amplitude that $\nu_{\tau}$ is produced at the source and oscillates to $\nu_{e}$ (remains $\nu_{\tau}$ ). Note that, as in the two-flavor case, the special condition $\theta+\psi=\mathrm{n} \pi$ kills the oscillation amplitudes, and in the $\mu \rightarrow \tau$ case there is a second condition when this can happen. The lack of symmetry between $P_{\mu \rightarrow e}$ and $P_{\mu \rightarrow \tau}$ results from our neglect of the $K_{21}$ coefficient in the amplitudes. Figure 5 shows the effect of choosing different $\mathrm{x}$ values (i.e. different $\Delta m^{2}$ values for fixed L/E) and non-zero $\psi_{\mu}$ values on the $P_{\mu \rightarrow e}=10^{-4}$ boundary in the $t a n^{2} \theta_{13}$ vs. $\tan ^{2} \theta_{23}$ plane, which is appropriate for the MiniBooNE [35] parameters.

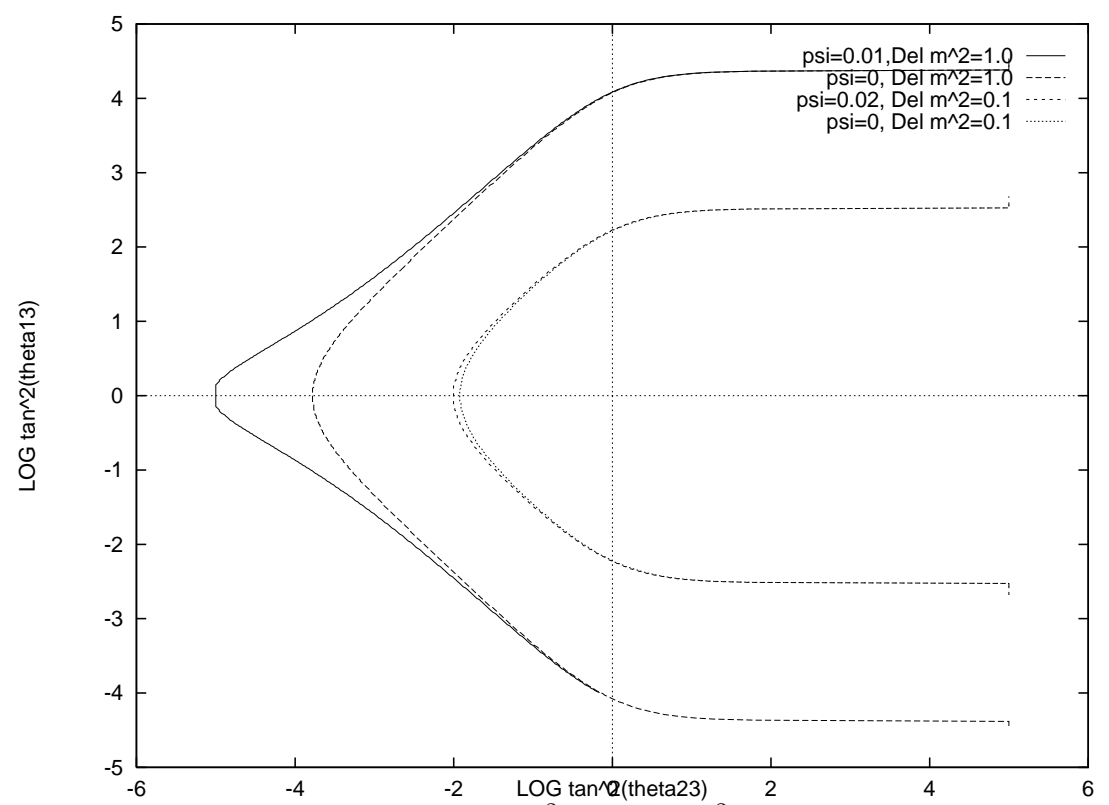

FIG. 5. Contours of $90 \%$ C.L. upper bounds in $\tan ^{2} \theta_{13}$ vs. $\tan ^{2} \theta_{23}$ space for several sets of $\psi_{\mu}, \Delta m^{2}$ values and with fixed value $P_{\mu \rightarrow e}=10^{-4}$. The new physics effects are taken to be at the source only.

For $\Delta m^{2}=1 e V^{2}$ the change in the small $\tan ^{2} \theta_{23}$ region as $\psi_{\mu}$ is turned on is noticeable already at $\psi_{m u}=0.01$. The distortion grows rapidly with $\psi$, and the $\operatorname{LOGtan}^{2} \theta_{23}$ minimum value moves well below $10^{-6}$ when $\psi_{\mu}=0.02$ and $\Delta m^{2}=1$. The symmetry of the graph about the $\tan ^{2} \theta_{13}=1$ line is a consequence of the equivalence of the $\theta$ values below and above $\pi / 4$ caused by the $\sin ^{2} 2 \theta$ factor.

The influence of flavor violations in $\tau$-flavor processes is not so tightly constrained experimentally as for strictly e-plus- $\mu$, and we look at this situation next. We contrast the bounds on $\tau$-appearance probability in the cases that the flavor violation is at the source alone and the source and detector both. In Fig. 6 we again look at the $\tan ^{2} \theta_{13}$ and $\tan ^{2} \theta_{23}$ plane boundaries for $P_{\mu \rightarrow \tau}=6 \times 10^{-4}$, as in the NOMAD experimental boundary, discussed in IV.A.1 above in the two-flavor model. Plots with $\Delta \mathrm{m}^{2}=9,6$ and $3 \mathrm{eV}^{2}$ are shown, with oscillation only in the 9 and $6 \mathrm{eV}$ cases but with $\psi_{\mu}=0.02,0.0$ and -0.02 for the source-only case at $\Delta m^{2}=3 e V^{2}$ and, for comparison, $\psi_{\mu}=0.01$ and -0.01 in the source-plus-detector case at $\Delta \mathrm{m}^{2}=3 \mathrm{eV}^{2}$. 


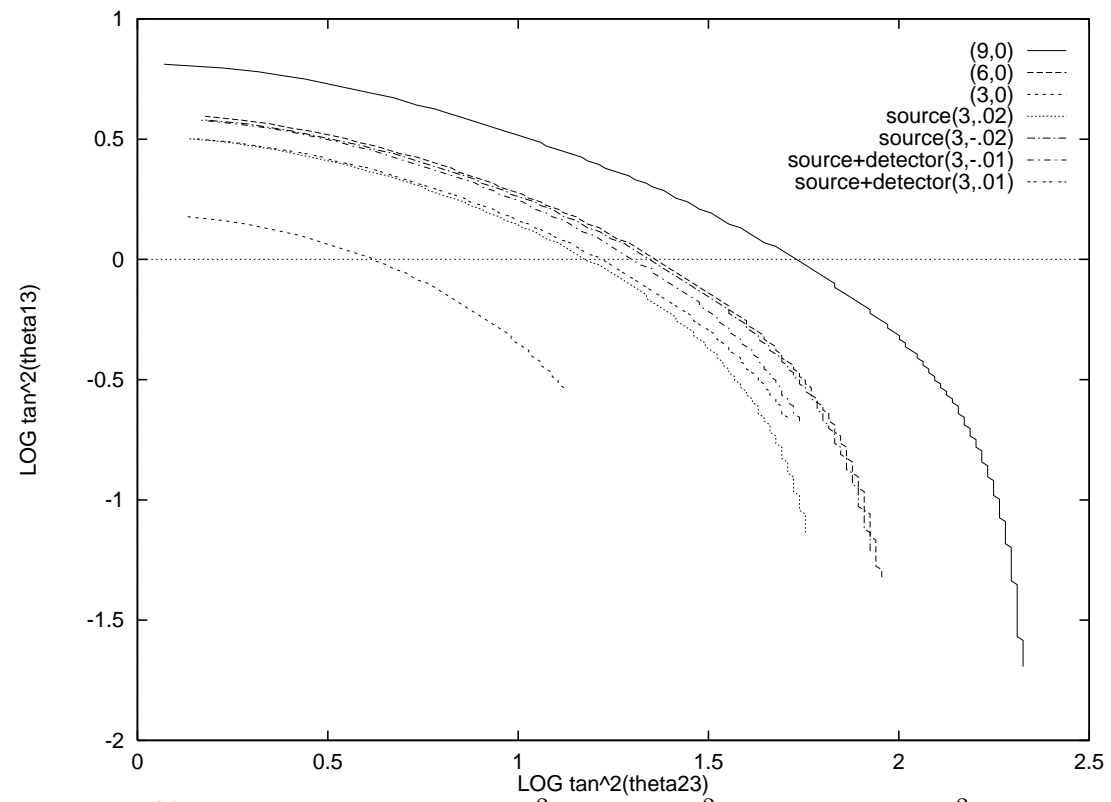

FIG. 6. Contours of $90 \%$ C.L. upper bounds in $\tan ^{2} \theta_{13}$ vs. $\tan ^{2} \theta_{23}$ space for $\Delta m^{2}=9,6$ and $3 e V^{2}$. The curves are designated as source or source and detector according to whether flavor violation is included at the source only or both the source and the detector. In parethesis the first number is the $\Delta m^{2}$ in $e V^{2}$ and the second is the value of $\psi_{\mu}$. For $\psi=0$ there is no direct flavor violation, so the "source" and "detector" labels are not meaningful. $P_{\mu \rightarrow \tau}=0.0006$, appropriate for NOMAD.

The most noteworthy feature of Fig. 6 is that there is a "confusion effect". The boundary curves for $\Delta m^{2}=3$ and 6 $\mathrm{eV}^{2}$ for different flavor violation situations crowd together and give a portrayal of the complications that arise when direct flavor violations and oscillations become competitive. The smaller values of $\psi$ used in the "source-plus-detector" situation compared to the "source-only" are forced by the lack of solutions to the probability equation when $|\psi|$ is 0.02. The reason is that there is a leading term $4 \times \tan ^{2} \psi$, so that there is no way to obtain $\mathrm{P}=0.0006$ when the lead term is 0.0016 and the $\sin ^{2} x$ term is as small as it is in the NOMAD experiment because of the small L/E.

\section{B. $\mu$-Decay as the Neutrino Source}

With muon decay as the neutrino source, there are electron and muon neutrino appearance examples within this two-state system involving the flavor-violating coefficients $F_{V 2111}, F_{V 2212}$ and $F_{V 2112}$. We give general expressions for cases where $\nu_{e} \rightarrow \nu_{\mu}, \nu_{e} \rightarrow \nu_{\tau}, \nu_{\mu \rightarrow e}$, and $\nu_{\mu} \rightarrow \nu_{\tau}$ in Appendix A. Again restricting ourselves to a V-A form for the new interactions, the general form of the probability factor for $\mu \rightarrow e$ transition is

$$
\begin{gathered}
P_{\mu \rightarrow e}=\left|-2 i \sin x e^{-i x} \sin \theta \cos \theta F_{V 2211}+\left(1-2 i \sin x e^{-i x} \sin ^{2} \theta\right) F_{V 2111}\right|^{2}+ \\
\left|-2 i \sin x e^{-i x} \sin \theta \cos \theta F_{2212}+\left(1-2 i \sin x e^{-i x} \sin ^{2} \theta\right) F_{V 2112}\right|^{2} .
\end{gathered}
$$

The corresponding expression for $P_{e \rightarrow \mu}$ is obtained by the interchanges $F_{V 2111} \leftrightarrow F_{V 2212}$ and $\cos \theta \leftrightarrow \sin \theta$. As in the $\pi$-decay case, Fig.(1), unless phases of the F's are of order 1 they play an insignificant role in the probability factors and we take them to be zero in the following discussion. Referring to Table 1, we see that we may drop the $F_{V 2111}=\tan \psi_{e e}$ term since it is several orders of magnitude smaller than the other lepton number violating coefficients in $\mu$-decay. We take $F_{V 2211}=1$ and expand the resulting expressions using the parameterizations $F_{V 2112}=\tan \psi_{e \mu}$ and $F_{V 2212}=\tan \psi_{\mu \mu}$. The resulting expressions are given by

$$
P_{\mu \rightarrow e}=\tan ^{2} \psi_{e \mu}+\sin ^{2} x \sin ^{2} 2 \theta\left(1+\tan ^{2} \psi_{\mu \mu}-\tan ^{2} \psi_{e \mu}-2 \cot 2 \theta \tan \psi_{\mu \mu} \tan \psi_{e \mu}\right)
$$

and

$$
\left.P_{e \rightarrow \mu}=\tan ^{2} \psi_{\mu \mu}+\left(\sin ^{2} x \sin 2 \theta \sin 2\left(\theta+\psi_{\mu \mu}\right)\right) \cos ^{-2} \psi_{\mu \mu}+\tan ^{2} \psi_{e \mu}\left(1-\sin ^{2} x \sin ^{2} 2 \theta\right)\right) .
$$

Dropping the $\psi_{e e}$ in the expressions for $P_{\mu \rightarrow e}$ and $P_{e \rightarrow \mu}$ produces the lack of symmetry between the two equations.

It is clear from Eq.(27) and the restriction $\left|F_{V 2112}\right| \equiv \mid$ tan $\psi_{e \mu} \mid \leq 8 \times 0.003=0.024$ that the effect of direct interactions on the amplitude of $\mu \rightarrow e$ oscillation will be important only if it turns out that $P_{\mu \rightarrow e} \leq 5 \times 10^{-4}$ and experiments 
can explore that region [37]. We return to this issue below. The $e \rightarrow \mu$ oscillation amplitude can be more strongly affected, because the branching fraction for $\mu \rightarrow e+\nu_{\mu}+\overline{\nu_{\mu}}$ is not directly constrained by bounds on isospin related, purely charged-lepton processes. Therefore, on these grounds alone, sizeable effects cannot be excluded in the $\nu_{e} \rightarrow \nu_{\mu}$ appearance case. The L/E dependence of $P_{e \rightarrow \mu}$ in Fig. 7 demonstrates the strong effect that the $\psi$ value has on the amplitude.

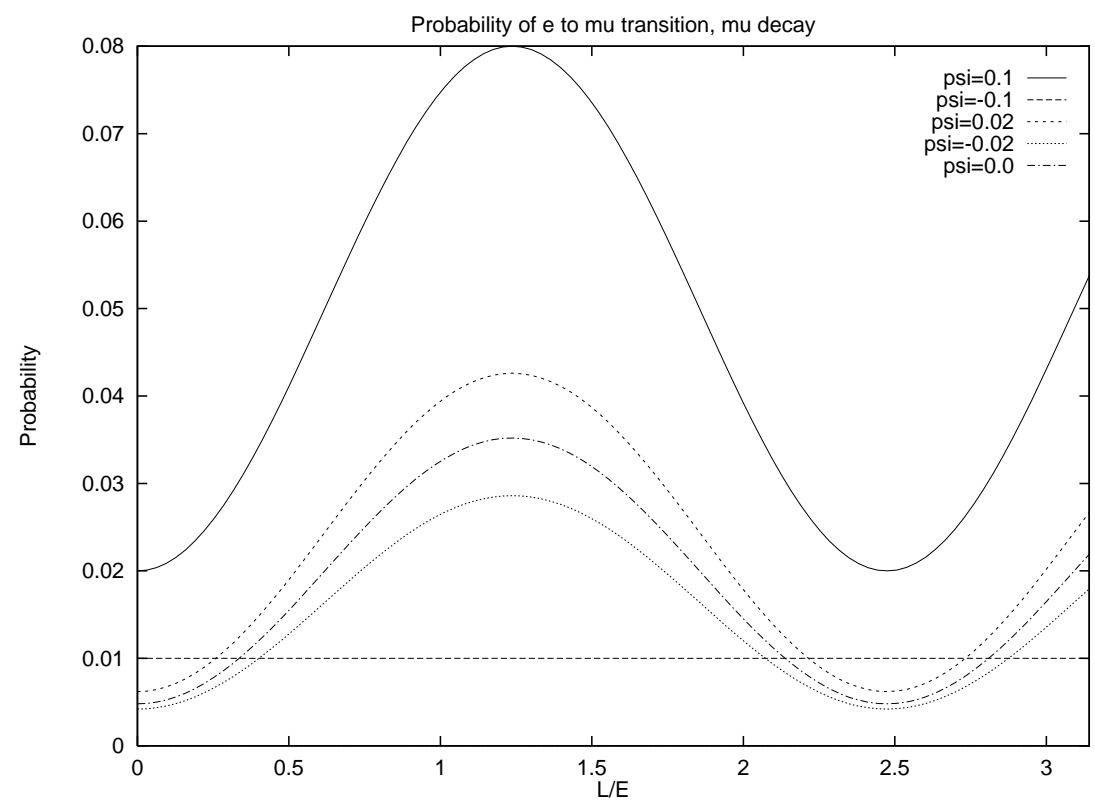

FIG. 7. Dependence of $P_{e \rightarrow \mu}$ for a $\mu$-decay source on L/E for $\theta=0.1, \psi_{e \mu}=0.0$ and several different values of $\psi_{\mu \mu}$ and with $\Delta m^{2}=1 e V^{2}$.

Here we show the oscillation probability as a function of $\mathrm{L} / \mathrm{E}$ for several choices of $\psi_{\mu \mu}$ with $\psi_{e \mu}=0$ and $\theta=0.1$. The effects of $\psi_{e \mu}$ are negligible with the chosen values of $\psi_{\mu \mu}$ and $\theta$ in this case also, as one anticipates from inspection of Eq.(29) and the bound on $\tan \psi_{e \mu}$ mentioned above. Note that the factor $\sin 2\left(\theta+\psi_{\mu \mu}\right)$ in Eq.(29) kills the oscillations in the case $\psi=-0.1$, simply because the choice $\theta=0.01$ is made for the graph.

\section{Comparison of $\nu_{e}$ Appearance to $\nu_{\mu}$ Appearance}

The behavior of $P_{e \rightarrow \mu}$ shown in Fig. 7 can translate into significant differences in the appearance probability for e compared to $\mu$ in neutrino experiments whose beams are extracted from muon collider storage rings, for example. In Fig. 8 we show the ratio of $P_{\mu \rightarrow e}$ to $P_{e \rightarrow \mu}$ as a function of $\psi_{\mu \mu}$, with $\theta=0.003$ and $\Delta m^{2}=1 e V^{2}$, chosen to be in a range allowed by LSND and not excluded by other experiments.

\footnotetext{
${ }^{5}$ In specific models, indirect constraints on $F_{V 2212}=\tan \psi_{\mu \mu}$ from $\mu \rightarrow e \gamma$ limits apply to a combination of amplitudes that includes a $\tan \psi_{\mu \mu}$.
} 


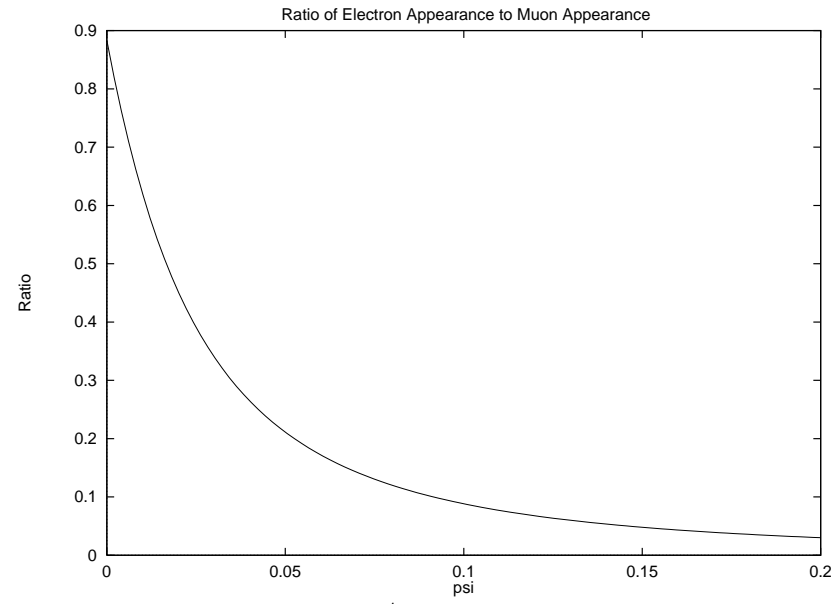

FIG. 8. Dependence of the ratio $P_{\mu \rightarrow e} / P_{e \rightarrow \mu}$ for a $\mu$ - decay source on $\psi_{\mu \mu}$ for $\sin ^{2} 2 \theta=0.003$, and with $\Delta m^{2}=1 e v^{2}$. See text for treatment of $\mathrm{L} / \mathrm{E}$.

The parameters chosen for Fig. 8 are guided by a recent proposal for a medium baseline appearance search with $\nu$ beams from $\mu$ decay [37. Below we summarize the relevant parameter values.

\begin{tabular}{|l|l|l|r|}
\hline $\sin ^{2} 2 \theta=0$ & $\langle L / E\rangle$ & $\sigma$ & $\sin ^{2} 2 \theta=1$ \\
\hline$\nu_{\mu}$ events $=11600 \mu^{-}$ & 0.66 & 0.20 & $\nu_{e}$ events $=6400 e^{-}$ \\
$\frac{\nu_{e}}{\nu^{\prime}}$ events $=5070 e^{+}$ & 0.75 & 0.26 & $\frac{\nu_{\mu}}{\text { events }=3280 \mu^{+}}$ \\
\hline
\end{tabular}

As Fig. 8 shows, the value of the ratio changes rapidly as a function of $\psi$, and offers a possible method to directly constrain $\psi$ down to 0.01 or less by comparing the $\nu_{\mu} \rightarrow \nu_{e}$ oscillation signal to its inverse. We can put this another way by comparing estimates of the number of $e^{-}$events with the number of $\mu^{+}$events detected downstream from $\mu^{-}$'s decaying in flight. In a purely oscillation picture, the $e^{-}$result from $\nu_{\mu}$ oscillating to $\nu_{e}$, while the $\mu^{+}$result from $\overline{\nu_{e}}$ oscillating to $\overline{\nu_{\mu}}$. In Fig. 9 we show a plot of the number of events expected vs. $\psi$ for the $\sin ^{2} 2 \theta$ and $\Delta m^{2}$ values assumed, given the SM event rates estimated in the search experiment proposed in [37].

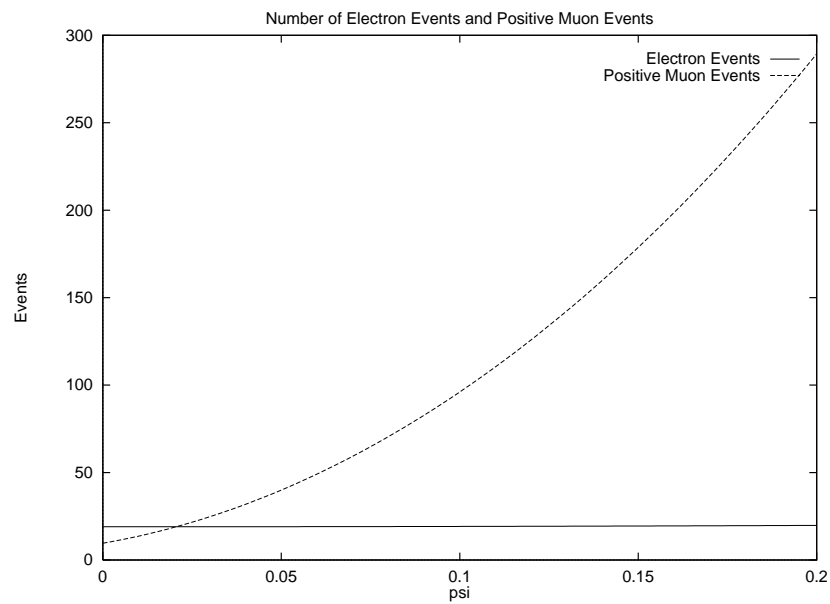

FIG. 9. The numbers of $e^{-}$and $\mu^{+}$appearance events as a function of $\psi_{\mu \mu}, \psi_{e \mu}=0$, for $\sin ^{2} 2 \theta=0.003$, and with $\Delta m^{2}=1 e V^{2}$. See text for treatment of $\mathrm{L} / \mathrm{E}$.

Figure 9. makes evident that interference between the oscillation and direct amplitudes greatly enhances the $\mu^{+}$ appearance rate estimate as $\psi$ grows, while there is little effect (a factor $\sec ^{2} \psi_{\mu \mu}$ ) on the $e^{-}$rate. The numbers for $\psi=0$ correspond to the pure oscillation numbers given in [37], but decreased by the factor $\sin ^{2} 2 \theta=0.003$ for $\Delta m^{2}=1 e v^{2}$, since the numbers there refer to $\sin ^{2} 2 \theta=1$. We see again that looking for new physics may be quite fruitful in the comparisons of appearance signals in "pure" neutrino beams provided by muon colliders. The current direct limit on $\psi_{\mu \mu}$ can be extracted from the limit [39] $\sigma\left(\overline{\nu_{\mu}}+e^{-} \rightarrow \mu^{-}+\bar{\nu}\right) / \sigma\left(\nu_{\mu}+e^{-} \rightarrow \mu^{-}+\nu\right)=t a n^{2} \psi \leq 0.05$, or $\tan \psi \leq 0.22$. As Figs. 8 and 9 indicate, finding $N_{e^{-}} \geq N_{\mu^{+}}$is sufficient to improve the bound roughly to $\psi \leq 0.02$, an order of magnitude better than the bound inferred from $[39]$. Allowing $\left|\psi_{e \mu}\right|=0.025$ has little effect on this statement. 


\section{Impact on Boundaries in e- $\mu$ Mixing Space}

Referring next to Eq.(28), let us consider what a proposed reach to $P_{\mu \rightarrow e} \leq 3.5 \times 10^{-4}$ at 90\% C.L. 37] affects the impact of direct interactions on the $\Delta m^{2}$ vs. $\sin ^{2} 2 \theta$ contour. Specifically, how do $\mu^{-} \rightarrow e^{-} \nu_{\mu} \overline{\nu_{\mu}}$ and $\rightarrow e^{-} \nu_{e} \overline{\nu_{\mu}}$ sources affect $\nu_{e}$ appearance from $\mu^{-}$sources (or $\overline{\nu_{e}}$ appearance from $\mu^{+}$sources). From (28) one can show that $\tan ^{2} \psi_{e \mu} \leq P_{\mu \rightarrow e} /\left(1-\tan ^{2} \psi_{\mu \mu} \sin ^{2} x\right)$, which essentially means that $\tan ^{2} \psi_{e \mu} \leq P_{\mu \rightarrow e}$ for small tan $\psi_{\mu \mu}$. In particular for $\left|\tan \psi_{\mu \mu}\right| \leq 0.2$ and $P_{\mu \rightarrow e}=3.5 \times 10^{-4}, 0.0187 \leq\left(\tan \psi_{e \mu}\right)_{\max } \leq 0.0193$. This range is slightly less than the modelindependent estimate, albeit on the generous side, of the bound $\left|\psi_{e \mu}\right| \leq 0.024$ that follows from the experimental limit on muonium-antimuonium transition [17].

We show the $P_{\mu \rightarrow e}=3.5 \times 10^{-4}$ contour in Fig. 10 with several sets of values of $\psi_{\mu \mu}$ and $\psi_{e \mu}$.

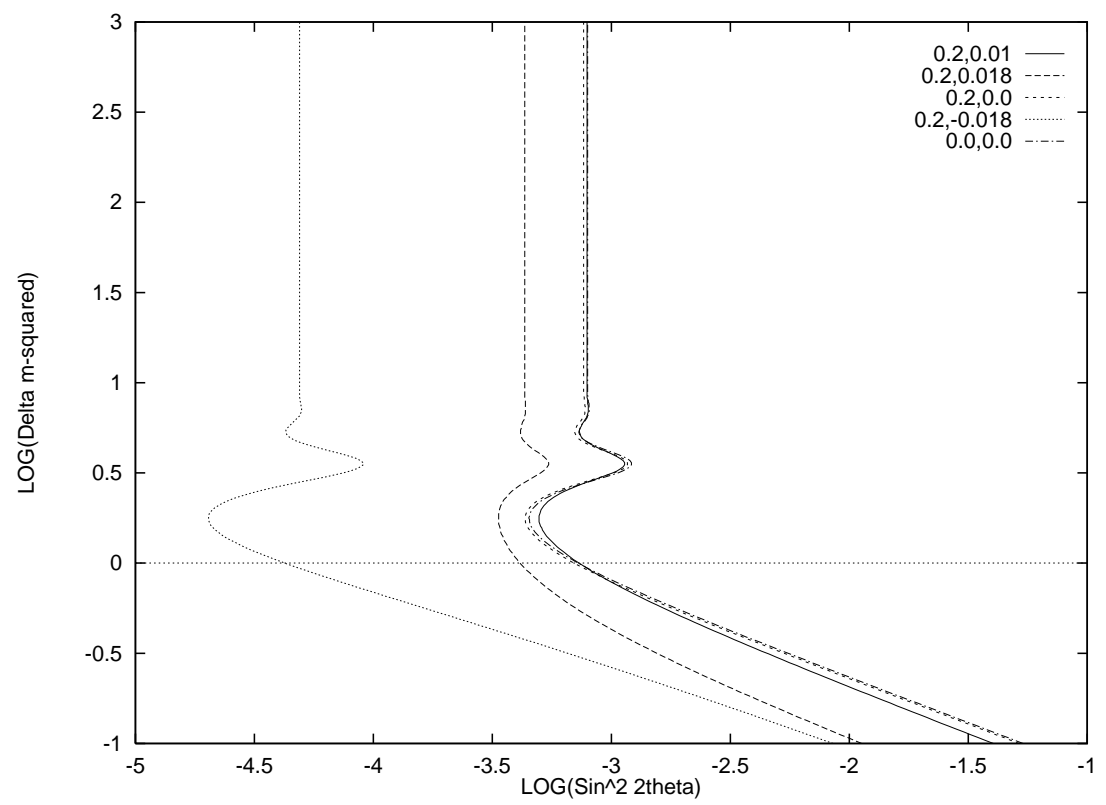

FIG. 10. $P_{\mu \rightarrow e}=3.5 \times 10^{-4}$ boundaries at $90 \%$ C.L. for several sets of $\psi_{\mu \mu}, \psi_{e \mu}$ values as shown on the legend.

The lack of sensitivity to $\psi_{\mu \mu}$ with $\psi_{e \mu} \simeq 0$, already evident from Eq.(28) and Fig. 9 is indicated in Fig. 10 by the pile-up of curves with $\psi_{e \mu} \leq 0.01$. The situation changes drastically when $\psi=0.018$, near its upper bound for solutions for $P=3.5 \times 10^{-4}$ to exist for some $\Delta m^{2}, \theta$ range of values. As noted in the introduction, possible direct interaction contributions are too small to affect the LSND oscillation signal fits. If LSND results are not confirmed and the limits drop to the level of $P_{\mu \rightarrow e} \approx 10^{-4}$, then the interpretation of those bounds should include the possible range of direct interaction strengths allowed by limits from other experiments. Correlated limits on $\Delta m^{2}, \sin ^{2} 2 \theta$ and $\tan \theta_{l l^{\prime}}$ can then be studied with the new data.

\section{SUMMARY OF RESULTS}

Direct lepton number violating interactions and neutrino mass terms often go hand-in-hand in physics beyond the standard model. With this in mind we developed a general parametrization of lepton number violating interactions that fits smoothly with the usual description of neutrino oscillations in terms of mixing angles. We defined generalized "probability factors" and illustrated with a number of examples drawn from accelerator appearance experimental setups. We concentrated on the case where the structure of the effective four-fermion, charged current, flavor-violating operators is $\mathrm{V}$-A. This is the cleanest situation and lends itself to a parameterization in terms of angles that makes the role of new interactions in the "wrong-flavor" appearance probability factors rather transparent. For example, in Eq.(23) the direct effects are expressed in terms of a leading term that gives all of the appearance probability when there is no oscillation, a second term that gives the interplay between the oscillation and direct effects and shows that there is a special condition $\theta-\psi=$ integer $\times \pi / 2$ where the usual oscillation term can be zero even when there is a neutrino mass difference and a mixing between flavors. The role of the CP-violating phase in the flavor-violating coupling is isolated in the last term, which is zero when the phase is a multiple of $\pi$, so the entire expression can be decomposed into readily interpretable pieces. 
Because the limits on lepton flavor violation are generally tight, the impact on the oscillation picture of appearance and disappearance is limited to cases where the oscillation probabilities are small and sensitive to the precise values of the mixing angles. We illustrate such situations in detail in Sec. 4, where the sensitive dependence of the appearance probability on the relative values of the flavor-violating angle $\psi$ and the mass-mixing angle $\theta$ are shown in Figs. 1,2 and 7 for the amplitude of the oscillation behavior, are shown in Fig. 3 for the the NOMAD, $\mu \rightarrow \tau$, two-flavor mixing boundaries and are shown in Fig. 6 for the corresponding three-flavor mixing boundaries in the one-mass-difference dominance model. The NOMAD experiment examples are carefully done within the limits imposed on the size of the flavor violations, as summarized in Table 1. We present similar considerations for the case where the source is $\mu$-decay, and the boundaries appropriate to a proposed $\mu$ storage ring environment are shown in Fig. 10, again for realistic constraints on the flavor-violation strength.

Fig. 4 illustrates another new effect that shows that care must be taken in interpreting limits set by wrong-flavor appearance searches. The behavior of the $P_{\mu \rightarrow e}=10^{-4}$ boundaries for source-only and source-plus-detector are quite different as viewed in the $\theta-\psi$ plane. The former boundary is "tilted" and so the bound on $|\psi|$ set by the $90 \%$ C.L. boundary is correlated with the value of $\theta$ so that, contrary to the usual expectation, the bound on $\psi$ is not obtained by setting $\theta=0$. The boundary for the latter situation, however, does conform to the usual expectation.

We show how the comparison of electron appearance and muon appearance experiments in a clean $\mu$ storage ring environment could be used to increase the sensitivity to direct, neutrino flavor violations by an order of magnitude in the discussion of Figs 8 and 9. These show the rather strong sensitivity of the muon appearance effects on the value of the violation parameter $\psi_{\mu \mu}$.

\section{CONCLUDING REMARKS}

A theme that recurs throught the analysis in Sec.4 is that small, direct flavor violation can seriously complicate the picture of wrong-flavor appearance in a number of experimentally realistic situations. In short, the interpretation of a signal can be quite ambiguous. To sort out the complete picture, a number of measurements at various values of $\mathrm{L} / \mathrm{E}$ in a variety of different channels is needed. Perhaps the cleanest and most flexible environment for such studies is provided by a $\mu^{+} \mu^{-}$collider with an associated facility for neutrino beams . Studies such as those exemplified in [37] can put much more stringent tests on direct neutrino flavor violation than currently exist, while at the same time allowing detailed oscillation analysis. Direct flavor-violation effects in semileptonic processes require pion and kaon beams of course, and the BooNE experiment will provide exploration of a wide range of parameter space, for example.

Though the direct flavor-violating strengths are constrained to be small, the matter-enhancement effects can lead to large transition probabilities, as analyzed in [25] and [26], and for small probabilities the "vacuum" effects in accelerator experiments can lead to important modification of the oscillation description. We conclude that the crucial role neutrinos play in our understanding of particle and astrophysics requires that data be analyzed with the

relevant flavor violation included to properly interpret current and future experiments. The present work provides a framework for that task and provides vivid, realistic examples of its application.

Acknowledgements: We thank Tom Weiler for discussions and John Ralston for suggestions on the manuscript. This work was supported in part by U.S. DOE Grant No. DE-FGO2-85ER40214. L.M.J. thanks the Department of Physics at Drury University for support during the course of this work.

\section{APPENDIX A: MUON DECAY APPEARANCE PROBABILITIES}

We gather the general formulas for appearance probabilities that apply when muon decay is the source of neutrinos. The presence of two neutrinos in the final state, only one of which is detected by a choice of lepton-flavor sensitive detector, makes the analysis slightly different from the situation when meson decay provides the source of neutrinos.

$$
\begin{aligned}
& P_{e \rightarrow \mu}=\sum_{k}\left|F_{2 k 1 j} U_{j c}^{*} e^{-i E_{c} t} U_{1 c}\right|^{2} . \\
& P_{\mu \rightarrow e}=\sum_{k}\left|F_{2 j 1 k}^{*} U_{j c} e^{-E_{c} t} U_{1 c}\right|^{2} . \\
& P_{e \rightarrow \tau}=\sum_{k}\left|F_{2 j 1 k} U_{j c}^{*} e^{-i E_{c} t} U_{3 c}\right|^{2} .
\end{aligned}
$$




$$
P_{\mu \rightarrow \tau}=\sum_{k}\left|F_{2 j 1 k}^{*} U_{j c} e^{-i E_{c} t} U_{3 c}\right|^{2} .
$$

In all of the expressions, the repeated indices within the absolute-square are understood to be summed. By choosing a given process, keeping the dominant flavor-transition terms and parameterizing the $\mathrm{F}$ amplitudes by angles, the $\mu$ decay source expressions in the text can be reproduced from these expressions.

[1] Super-Kamiokande Collaboration, Y. Fukuda et al., Phys. Rev. Lett. 81, 1158 (1998), hep-ph/9807003.

[2] P. Langacker, Nucl. Phys. (Proc. Suppl.) 77, 241 (1999), hep-ph/9811460.

[3] C. Cardall and G. Fuller, Phys. Rev. D 53, 4421 (1996).

[4] T. Ohlsson and H. Snellman, hep-ph/9903252.

[5] V. Barger, S. Pakvasa, T. Weiler, and K. Whisnant, Phys. Rev. D 58, 093016 (1998), hep-ph/9806328.

[6] P. Langacker, Phys. Rev. D 58, 093017 (1998), hep-ph/9805281, presents an argument that efficient oscillation between standard model and sterile neutrinos can occur naturally in a class of string models.

[7] D. McKay and H. Munczek, Phys. Rev D, 19, 985 (1979).

[8] A. Zee, Phys. Lett. 93B, 389 (1980).

[9] K.S. Babu, Phys. Lett. B203 (1988).

[10] N. Gaur, A. Ghosal, E. Ma, and P. Roy, Phys. Rev. D, 071301 (1998), hep-ph/9806272.

[11] J. Valle, Prog. Part. Nucl. Phys. 40, 43-54 (1998), hep-ph/9711374.

[12] D. Wyler and L. Wolfenstein, Nucl. Phys. B218, 205 (1983).

[13] L. Johnson and D. McKay, Phys. Lett. B 433, 355 (1998).

[14] C. Athanassopoulos et al., Phys. Rev. C 54, 2685 (1996).

[15] C. Athanassopoulos et al., Phys. Rev. Lett. 81, 1774 (1998).

[16] K. Eitel and B. Zeitnitz for the Karmen Collaboration, Nucl. Phys. Proc. Suppl. 77, 212-219 (1999), hep-ex/9809007.

[17] L.Willmann et al., Phys. Rev. Lett. 82, 49 (1999).

[18] S. Bergmann and Y. Grossman, Phys. Rev. D 59, 093005 (1999), hep-ph/9809524.

[19] NOMAD Collaboration, P. Astier et al., Phys. Lett. B 453, 169 (1999).

[20] S. Bergmann, Nucl. Phys. B515, 368 (1998), hep-ph/9707398.

[21] B.T. Cleveland et al., Nucl. Phys. B (Proc. Suppl.) 38, 47 (1995).

[22] GALLEX collaboration, W. Hampel et al., Phys. Lett. B388, 384 (1996).

[23] SAGE collaboration, J. Abdurashitov et al., Phys. Rev. Lett. 77, 4708 (1996).

[24] Kamiokande collaboration, Y. Fukuda et al., Phys. Rev. Let, 77, 1683 (1996); Super-Kamiokande collaboration, Y. Suzuki, presented at Neutrino-98, Takayama, Japan, June 1998.

[25] M. Gonzalez-Garcia et al., Phys. Rev. Lett. 82, 3202 (1999), hep-ph/9809531.

[26] N. Fornengo, M. Gonzalez-Garcia and J. Valle, hep-ph/9906539.

[27] V. Barger, J. Learned, S. Pakvasa and T. Weiler, Phys. Rev. Lett. 82, 2640 (1999), astro-ph/9810121.

[28] P. Lipari and M. Lusignoli, Phys. Rev. D 60, 013003 (1999), hep-ph/9901350.

[29] V. Barger, J. Learned, P. Lipari, M. Lusignoli, S. Pakvasa, and T. Weiler, hep-ph/9907421.

[30] P. Langacker and D. London, Phys. Rev. D 39, 266 (1989).

[31] C. Giunti, C.W. Kim and U.W. Lee, Phys. Rev. D 45, 2414 (1992).

[32] Review of Particle Properties, C. Caso et al., Eur. Phys. J. C 3, 1 (1998).

[33] J. Kim, P. Ko and D.-G. Lee, Phys. Rev. D 56, 100 (1997).

[34] M. Brhlik, G. Good, and G. Kane, Phys. Rev. D 59, 115005 (1999) and "Large Mixing and CP Violation in Neutrino Oscillations", Zhi-zhong Xing, hep-ph/9908381 are recent examples of studies giving large CP-violating angle solutions.

[35] "MiniBooNE: the Booster Neutrino Experiment" A. Bazarko for the Booster Neutrino Experiment, hep-ex/9906003.

[36] "Conceptual Study of an "Anti-Tagged" Experiment Searching for $\nu_{\mu} \rightarrow \nu_{e}$ Oscillation", L. Ludovici and P. Zucchelli, CERN-PPE/96-181.

[37] "A Medium Baseline Search for $\nu_{\mu} \rightarrow \nu_{e}$ Oscillations at a $\nu$ Beam Facility from Muon Decays", A. Bueno, M. Campanelli and A. Rubbia, CERN-EP/98-140, Mod. Phys. Lett. A, to be published.

[38] "Neutrino Oscillation Physics at Fermilab: The NuMI-MINOS Project", the MINOS Collaboration, NuMI-L-375 (May 11, 1998).

[39] CHARM Collaboration, F. Bergsma et al., Phys. Lett. 122B, 465 (1983). 